\title{
Green synthesis of silver nanoparticles using aqueous plant extracts and its application as optical
} sensor

\begin{abstract}
The syntheses of metal nanoparticles have taken a new route from the emergence of green chemistry. We propose the synthesis of silver nanoparticles through simple bio synthetic method. Metal nanoparticles prepared are characterized by TEM, UVVis and FTIR spectroscopy. TEM image show the nanoparticles are nearly spherical in shape with size about $40-50 \mathrm{~nm}$. UV-vis spectra show distinct peak of silver at around $427 \mathrm{~nm}$ corresponding to the surface plasmon peak of silver nanoparticles. The reduction in the surface plasmon peak of silver nanoparticles (Ag NPs) in addition to hydrogen peroxide at increasing concentrations supports our stand that it can be used as an optical sensor. Colorimetric detection of hydrogen peroxide is done with cellulose substrates.
\end{abstract}

Volume 2 Issue 3 - 2017

\author{
Subramanian L,' Sabu Thomas, ${ }^{2}$ Obey Koshy ${ }^{2}$ \\ 'Department of Chemistry, Amrita University, India \\ ${ }^{2}$ Mahatma Gandhi University, India
}

Correspondence: Obey Koshy, International and InterUniversity Centre for Nanoscience and Nanotechnology, Mahatma Gandhi University, PD Hills, Kottayam, Kerala, 686560, India, Email ObeyKoshy@gmail.com

Received: February 01, 2017| Published: April 03, 2017

\section{Introduction}

Metal nanoparticles have received a lot of attention in the recent decades due to their unique ability to alter optical, electrical, biological properties. ${ }^{1-5}$ Nanoparticles are also interesting because of their huge increase in surface area and a surge in conductivity compared to their metal counterparts. Moreover, metal nanoparticles also show surface plasmon resonance. These properties make them useful to many applications such as bio-sensing, imaging, drug delivery, HIV treatment, Cancer treatment, optical spectroscopy and Surface Enhanced Raman scattering (SERS). ${ }^{2-4}$

Amongst different type of metal nanoparticles Ag NPs (Silver nanoparticles) are of great interest due to their potent antimicrobial activity against different type of pathogens. ${ }^{6-10}$ This extends their application into medicine, wound healing, dental materials, coating of stainless steel material, water treatment, sunscreen lotions, elimination of microorganisms on textile fabrics, cosmetics, optical transparent composites, polymer composites etc. ${ }^{11-13}$ The smaller the particle, the greater the antimicrobial activity. However, these particles are very reactive and consequently form aggregates losing their fundamental properties. This leads to the phenomenon called agglomeration. To deal with this challenge passivating agents such as polyvinylpyrrolidone(PVP), poly vinyl alcohol(PVA), hyper branched polyurethane $(\mathrm{HP})$ and polyacronitrile(PAN) with reducing agents such as sodium borohydride, hydrazine, glycerol, etc have been used successfully in the synthesis and stabilization of silver nanoparticles. ${ }^{14-16}$ The use of these passivating and reducing agents introduced the challenge of high cost and toxicity, thus threatening environmental sustainability and limiting the biological application of these noble materials. Physical methods require highly sophiscated instruments and is of high cost. This crisis can be solved by using eco friendly, environmental benign methods for the synthesis of AgNPs. This eventually led to the entry of green methods for synthesizing AgNPs. Thus, AgNPs are synthesized using varying types of plant extracts, green algaes, leaf broths etc. We report the use of aqueous leaf extracts of mangifera indica. They are natural mango leaves abundant in Indian subcontinent. ${ }^{17-21}$ They are largely available and free of cost. The chemical structure of mangifera indica do have $\mathrm{OH}$ groups which can get easily attached to Ag NPs thus forming Ag NPs. The leaf extract acts as both reducing and stabilizing agent. In other words it acts as a surfactant preventing agglomeration.

One of the potential application of Ag NPs is the determination of hydrogen peroxide. Hydrogen peroxide has great relevance pertaining to environmental, pharmaceutical and clinical research. ${ }^{22-24}$ Hydrogen peroxide is a very good oxidizing agent. In addition, hydrogen peroxide is a byproduct of number of enzymatic reaction involving glucose, cholesterol and lactate. However, higher concentration and amount of hydrogen peroxide can induce potentially serious disease in human body and can cause diseases such as cancer etc. The high concentration rates of hydrogen peroxide can lead to these problems. Thus a stable method for the sensing of different concentrations of hydrogen peroxide has to be developed. Many methods for the sensitive determination of hydrogen peroxide has been developed such as chemiluminescence, optical detection and electrochemical method. Of these, optical detection is found to be the best method as it is convenient and low cost. In the present manuscript, we synthesized silver nanoparticle by green route. The silver nanoparticle synthesized were characterized and coated on a cellulose substrate for optical detection of hydrogen peroxide.

\section{Experimental}

\section{Materials required}

Leaves of mangifera indica plucked from nearby trees, $\mathrm{AgNO}_{3}$ (Silver Nitrate) (Sigma Aldrich), Cellulose fibres (1\%) (University of Maine, U.S.A)

Synthesis techniques for Ag NPs: Leaves of mangifera indica were taken from a nearby tree. $10 \mathrm{gm}$ of mangifera indica leaves were taken. They were rinsed with deionized, double distilled water and cut into small pieces. They were boiled in $75 \mathrm{~mL}$ of deionized water for 10 minutes. The leaf broth was allowed to cool. Now, it was filtered out and secured. Whatman filter paper (Grade 1) was used for the filtration The leaf extract $(2,3,4,5,10,15,20 \mathrm{ml})$ was added to a vigorously stirred $30 \mathrm{ml}$ solution of $1 * 10^{-4} \mathrm{M} \mathrm{AgNO}_{3}$. Stirring continued for 30 min to get the colloids labelled c1 to c6. $100 \mathrm{ml} \mathrm{AgNO}_{3}$ was prepared using $\mathrm{H}_{2} \mathrm{O}$ as the solvent. 
Effect of temperature: To different concentrations of $\mathrm{AgNO}_{3}$ broth solution, the temperature was increased gradually from $30^{\circ} \mathrm{C}$ to $60^{\circ} \mathrm{C}$. The temperature was readily increased $30,40,45,50,60^{\circ} \mathrm{C}$ to the broth to $\mathrm{AgNO}_{3}$ solution having a concentration of about $0.012 \mathrm{~N}$. The effect of temperature on the synthesized Ag NPs was studied.

Effect of pH: The pH of the solution was changed and monitored. $\mathrm{NaOH}$ (Sodium Hydroxide) solid aqueous pellets were added to the $30 \mathrm{ml} \mathrm{H}_{2} \mathrm{O}$. This was added to $10 \mathrm{ml}$ nanoparticle in the solution having the broth concentration $0.012 \mathrm{~N}$. Thus the $\mathrm{pH}$ of the solution was changed from $8 \mathrm{pH}$ to $12 \mathrm{pH}$. The $\mathrm{pH}$ was steadily changed $(8,9$, $10,11,12 \mathrm{pH})$. Thus, the syntheses of nanoparticles were optimized by three methods namely
i. By changing the concentration of the broth
ii. By changing the temperature
iii. By changing the $\mathrm{pH}$ range of the colloidal solution.

Application of silver nanoparticles for hydrogen peroxide detection: The synthesis of silver nanoparticles are just the same as explained above using green, leaf extract of mangifera indica. Hydrogen peroxide present in laboratory was taken. Different concentrations of hydrogen peroxide was made from $1 \mathrm{M}$ to $1 \mu \mathrm{M}$.The concentrations includes $1 \mathrm{M}, 100 \mathrm{mM}, 10 \mathrm{mM}, 1 \mathrm{mM}, 100 \mu \mathrm{M}, 75 \mu \mathrm{M}$, $50 \mu \mathrm{M}, 25 \mu \mathrm{M}, 10 \mu \mathrm{M}$ and $1 \mu \mathrm{M}$. Hydrogen peroxide of different concentrations was added to the synthesized Ag NPs.

Cellulose coated silver nanoparticle: Cellulose fiber is coated with silver nanoparticle by drop casting method.

\section{Results and discussion}

Figure 1 shows UV-Vis spectra of silver nitrate, broth and silver nanoparticle prepared at different broth concentration. The spectra of silver nitrate and broth didn't show any distinct peak. However the distinct peaks are observed for broth reduced silver nitrate at around $427 \mathrm{~nm}$ corresponding to the surface plasmon peak of silver nanoparticles. As the broth concentration is reduced, two peaks are observed at $380 \mathrm{~nm}$ and $427 \mathrm{~nm}$. As the broth concentration increased, peak at $380 \mathrm{~nm}$ disappeared and merge with $427 \mathrm{~nm}$ peak corresponding to the plasmon peak of silver. ${ }^{1}$

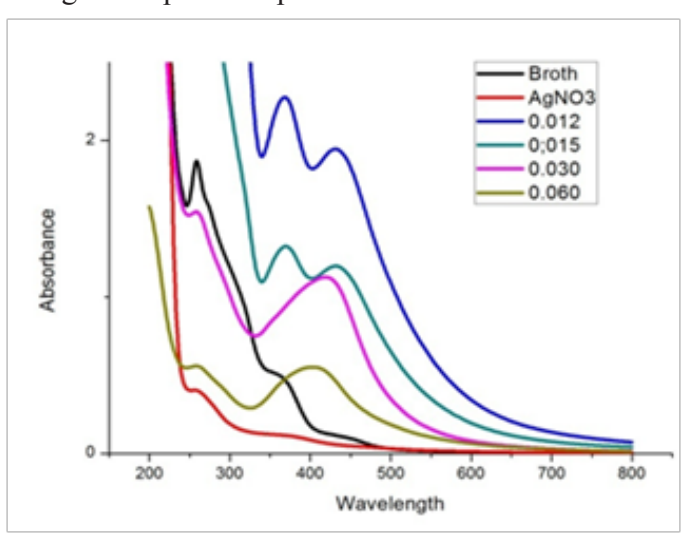

Figure I UV-Vis spectra of $\mathrm{AgNO}_{3}$, broth and silver nanoparticles prepared at different broth to $\mathrm{AgNO}_{3}$ concentration ratio's.a

Figure 2 shows the formation of silver nanoparticles at different temperature namely $30,40,45$ and $50^{\circ} \mathrm{C}$. Distinct peak at $427 \mathrm{~nm}$ is observed at temperature 30 and $40^{\circ} \mathrm{C}$. As the temperature is increased further, the sharpness of the peak decreased indicating the agglomeration of nanoparticles. The effect of $\mathrm{pH}$ on the nanoparticle formation is studied and showed in Figure 3. From Figure 3 obtained, it is found that the nanoparticles formed at $\mathrm{pH} 8$ were distinct and seems to be less agglomerated due to the sharpness of the peak. Nanoparticles were found to be get agglomerated above $\mathrm{pH} 8 .^{3}$ The FTIR peaks (Figure 4) at $3339.69 \mathrm{~cm}^{-1}$ shows the presence of $\mathrm{OH}$ groups and $1634.87 \mathrm{~cm}^{-1}, \mathrm{C}=0$ groups. ${ }^{4}$ Thus, the presence of $\mathrm{C}=\mathrm{O}$ groups and $\mathrm{OH}$ groups indicates that it can be attached to cellulose matrix having high concentration of hydroxyl group.

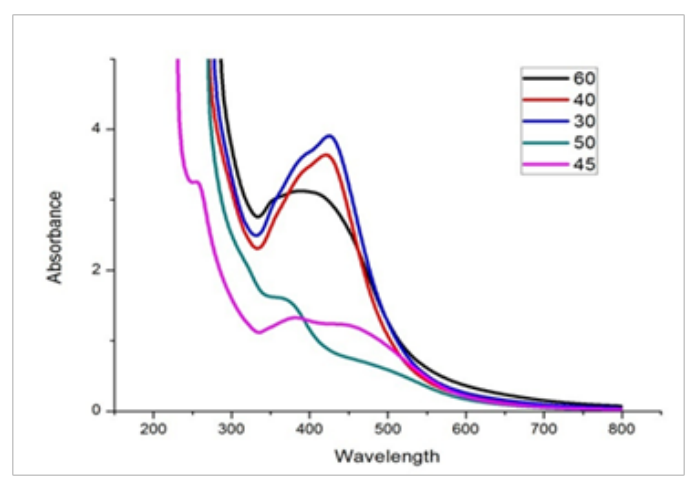

Figure 2 UV-Vis spectra of silver nanoparticles prepared at different temperatures.

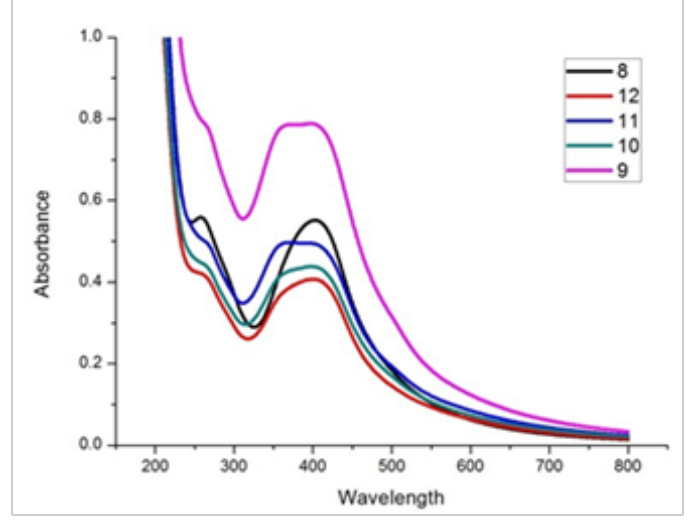

Figure $3 \mathrm{UV}$-Vis spectra of silver nanoparticles prepared at different $\mathrm{pH}$ values.

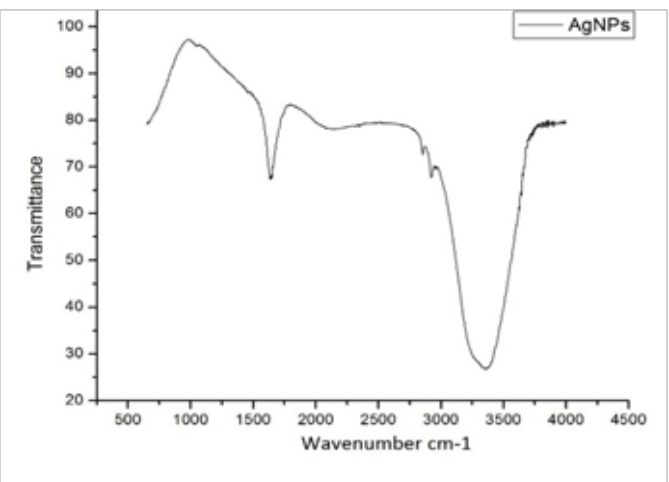

Figure 4 FTIR Spectra of the colloidal solution containing Ag NPs.

The formation of Ag NPs is quiet evident from the TEM image (Figure 5) obtained. Silver nanoparticles in the range having size $40-50 \mathrm{~nm}$ are observed in the image. The UV-vis data Figure 6 shows the detection of hydrogen peroxide using bio-synthesized silver nanoparticles. The UV-vis data shows constant sharp decline on increasing the concentration of hydrogen peroxide. The peak of Ag NPs steadily decreases upon having higher concentrations of 
$\mathrm{H}_{2} \mathrm{O}_{2}{ }^{22}$ This aptly defines the fact that the Ag NPs act as an optical sensor. Figure 7 shows the silver nanoparticle solution drop casted on cellulose substrates. Figure 7 shows that the cellulose substrate appears yellowish due to the low concentration of hydrogen peroxide. As the concentration is increased, the cellulose appeared to be white in color. So we can use cellulose for the detection of hydrogen peroxide. It can also be used to stabilize Ag NPs due to the presence of $\mathrm{OH}$ groups.

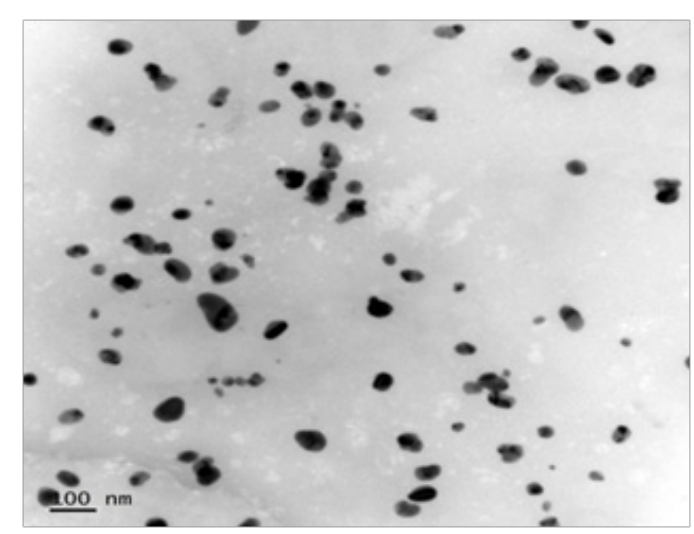

Figure 5 TEM image of Ag NPs.

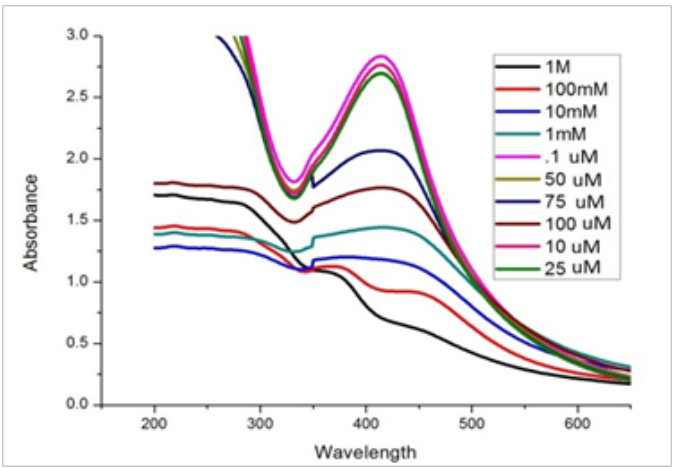

Figure 6 UV-vis spectra of Ag NPs towards different concentrations of $\mathrm{H}_{2} \mathrm{O}_{2}$.

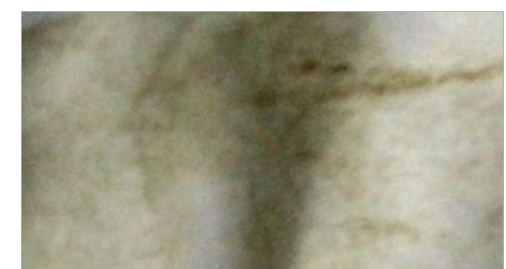

(A)

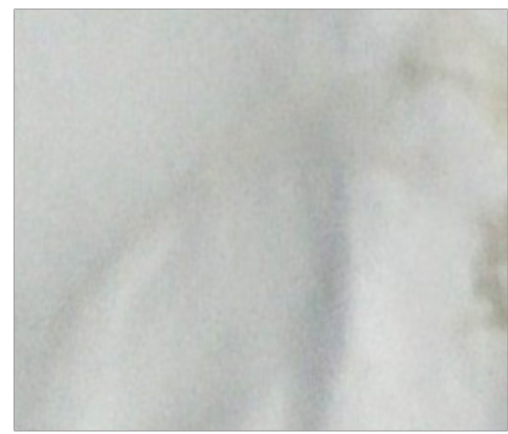

(B)

Figure 7 Cellulose coated with silver nanoparticles to different concentration of hydrogen peroxide.

A) $2 \times 10^{-6} \mathrm{M}$; B) $2 \times 0.01 \mathrm{M}$.

\section{Conclusion}

A simple colorimetric detection method for hydrogen peroxide is demonstrated. TEM image show the nanoparticles are nearly spherical in shape with size about $40-50 \mathrm{~nm}$. UV-vis spectra show distinct peak of silver at around $427 \mathrm{~nm}$ corresponding to the surface plasmon peak of silver nanoparticles. The reduction in the surface plasmon peak of Ag NPs on addition of hydrogen peroxide at increasing concentrations supports our stand that it can be used as an optical sensor. Colorimetric detection of hydrogen peroxide is done with cellulose substrates. Thus, Ag NPs can be synthesized easily using aqueous plant extracts which can act as surfactants. The shape of nanoparticles can also be controlled by changing the parameters like $\mathrm{pH}$, concentration of reducing agents etc. Paper based technique is developed for the detection of hydrogen peroxide.

\section{Acknowledgements}

None.

\section{Conflict of interest}

The author declares no conflict of interest.

\section{References}

1. Shiv Shanker, Akhilesh Rai, Absar Ahmad, et al. Rapid synthesis of $\mathrm{Au}, \mathrm{Ag}$, and bimetallic Au core-Ag shell nanoparticles using Neem (Azadirachta indica) leaf broth. J Colloid Interface Sci. 2004;275(2):496502.

2. Akshaya K Samal, Lakshminarayana Polvarapu, Sergio Rodal Cederia. Size tunable Au@Ag core-shell nanoparticles: synthesis and surfaceenhanced raman scattering properties. Langmuir. 2013;29(48):1507615082 .

3. Guang Yang, Jianjian Xie, Feng Hong. Antimicrobial activity of silver nanoparticle impregnated bacterial cellulose membrane: Effect of fermentation carbon sources of bacterial cellulose. Carbohydrate Polymers. 2012;87(1):839-845.

4. Sheny DS, Mathew J, Philip D. Phytosynthesis of Au, Ag and AuAg bimetallic nanoparticles using aqueous extract and dried leaf of Anacardium occidentale. Spectrochim Acta A Mol Biomol Spectrosc. 2011;79(1):254-262.

5. Shekhar Agnihotri, Soumyo Mukherji, Suparna Mukherji. Sizecontrolled silver nanoparticles synthesized over the range 5-100 nm using the same protocol and their antibacterial efficacy. RSC Advances. 2014;4(8):3974-3983.

6. Prakash P, Gnanaprakasam P, Emmanuel R, et al. Green synthesis of silver nanoparticles from leaf extract of Mimusops elengi, Linn. for enhanced antibacterial activity against multi drug resistant clinical isolates. Colloids Surf B Biointerfaces. 2013;108:255-259.

7. Rafie HM El, Zahran MK. Brown algae himantothallus grandifolius (Desmarestiales, Phaeophyceae) suppresses proliferation and promotes apoptosis-mediated cell death in tumor cells. Carbohydrate Polymers. 2013;96:403-410.

8. Ye Tian, Fengli Wang, Yanxia Liu. Green synthesis of silver nanoparticles on nitrogen-doped graphene for hydrogen peroxide detection. Electrochimica Acta. 2014;146:646-653.

9. Marimuthu Thiripura Sundari, Atmakuru Ramesh. Isolation and characterization of cellulose nanofibers from the aquatic weed water hyacinth-Eichhornia crassipes. Carbohydrate Polymers. 2012;87(2):1701-1705.

10. Tstomu Hirakawa, Prashant V Kamat. Charge separation and catalytic activity of $\mathrm{Ag} @ \mathrm{TiO}_{2}$ core-shell composite clusters under UVirradiation. J Am Chem Soc. 2005;127(11):3928-3934. 
11. Wojciech Czaja, Alima Krystynowicz, Stanslaw Bielecki, et al Microbial cellulose-the natural power to heal wounds. Biomaterials. 2006;27(2):145-151.

12. Jose Ruben Morones, Jose Luis Elechiguerra, Alejandra Camacho, et al. The bactericidal effect of silver nanoparticles. Nanotechnology 2005;16:2346-2353

13. Filippo E, Serra A, Manno D. Poly(vinyl alcohol) capped silver nanoparticles as localized surface plasmon resonance-based hydrogen peroxide sensor. Sensors and Actuators B. 2009;138(2):625-630.

14. Rajib Ghosh, Chaudhari, Sanatanu Paria. Core/shell nanoparticles: classes, properties, synthesis mechanisms, characterization, and applications. Chem Rev. 2012;112(4):2373-2433.

15. Aaron D Brumbaugh, Katelyn A Cohen, Sarah K St Angelo. Ultra small copper nanoparticles synthesized with a plant tea reducing agent. ACS Sustainable Chem Eng. 2014;2(8):1933-1939.

16. Jun Zhao, Domgming Zhang, Xiuli Zhang. Preparation and characterization of copper/silver bimetallic nanowires with core-shell structure. Surface Interface Analysis. 2015;47(44):529-534.

17. Dunying Deng, Yunxia Jin, Yuanrong Cheng. Copper nanoparticles aqueous phase synthesis and conductive films fabrication at low sintering temperature. ACS Applied Materials Interface. 2013;5(9):3839-3846.

18. Valentin V, Makarov, Svetlana S. et al. Biosynthesis of stable iron oxide nanoparticles in aqueous extracts of hordeum vulgare and rumex acetosa plants. Langmuir. 2014;30(20):305982-5988.
19. Lanlan Huang, Xiulan Weng, Zuliang Chen. Green synthesis of iron nanoparticles by various tea extracts: comparative study of the reactivity. Spectrochimica Acta A: Molecular and Bilomolecular Spectoscopy. 2014;130:295-301

20. Lanlan Huang, Xiulan Weng, Zuliang Chen. Synthesis of iron-based nanoparticles by green tea extract and their degradation of malachite. Industrial Crops and Products. 2013;51:342-347.

21. Lanlan Huang, Xiulan Weng, Zuliang Chen. Synthesis of iron-based nanoparticles using oolong tea extract for the degradation of malachite green. Spectrochimica Acta Part A: Molecular and Biomolecular Spectroscopy. 2014;117:801-804.

22. Nitinaivinij K, Parnklang $\mathrm{T}$, Thammacharoen C. Colorimetric determination of hydrogen peroxide by morphological decomposition of silver nanoprisms coupled with chromaticity analysis. Analytical Methods. 2014;6:9816-9824.

23. Wang $\mathrm{G}, \mathrm{Zhu} \mathrm{X}$, Jiao $\mathrm{H}$, et al. Ultrasensitive and dual functional colorimetric sensors for mercury (II) ions and hydrogen peroxide based on catalytic reduction property of silver nanoparticles. Biosensors and Bioelectronics. 2012;31(1):337-342.

24. Aadil KR, Barapatre A, Meena AS, et al. Hydrogen peroxide sensing and cytotoxicity activity of Acacia lignin stabilized silver nanoparticles International Journal of Biological Macromolecules. 2016;82:39-47. 